\title{
Determinación de actividad de acetilcolinesterasa (ACE) mediante biopsia rectal por succión
}

\author{
Drs. Guillermo Venegas V., ${ }^{1}$ Tatiana Rudloff F., ${ }^{2}$ Sra. Karin Reinecke ${ }^{3}$
}

\begin{abstract}
ASSESMEN'T OF ACETYLCHOLINESTERASE ACTIVITY BY MEANS RECTAL BIOPSY
Acety]cholinesterate activity was measured in rectal biopsy specimens obtained from 34 childrern, 12 patients with a previous surgical proxtdure for Hlirwhprong's disease and 22 jatients with chrone constipation. The method and the results are described.

It is suggested that the assay of acetylebolinesterase atcivity in rectia biopsy material is a siniple and iuick procedure useful in the diagnosis of ticongcnital intestiual ingunglionosis.
\end{abstract}

La Enfermedad de Hirschsprung o aganglionosis deriva de una ausencia congénita de los ganglios entéricos intramurales, siempre a partir del ano en dirección proximal y en segmentos variables, que

Proyecto 2.05.14. Financiado por Vicerrectoria de Investipaciôn, Universitad de Concepeión.

I Profesur Auxiliar Depto. Pediatria Facultad cle Medicina, Universidad de Concepticin.

2Médico Pediatra Sección Gastronterología, Servicio Pediatría Hospital Como. Grant B., Concepción.

${ }^{3}$ Bioxuinica, Docente Facultad de Ciencias Binlógicas, Universidad de Coorepcioin. pueden alcanzar todo el intestino. Por causas desconocidas, y entre las seis y doce semanas de vida intrauterina, los neuroblastos entéricos sufren una detención en alturas variables de su migración craneocaudal, configurando la longitud del segmento agangliónico y los posteriores síntomas clínicos. Al faltar la función moduladora e integradora de los plexos intramurales sobre el peristaltismo de la zona agangliónica, su motilidad es ineficaz para la propulsión, conduciendo a retención fecal progresiva, suboclusión mantenida y dilatación intestinal secundaria., $1,11,13$ 
El estudio del plexo de Auerbach mediante biopsia de las capas musculares intestinales, es desde 1948 un paso imprescindible en el diagnóstico de megacolon congénito, con resultados aceptables hasta el presente. Sin embargo, su realización exige anestesia general y hospitalización para resecar un fragmento apreciable de la pared intestinal o rectal, con potenciales peligros de perforación o estenosis residual., 3, 12

La biopsia rectal por succión (BKS), incluyendo exclusivamente mucosa y submucosa, se utiliza desde 1965, con el fin de observar el plexo de Meissner y obviar la mayoria de los inconvenientes de las biopsias de pared total. La BRS no es agresiva, no requiere hospitalización ni anestesia y carece prácticamente de complicaciones. ${ }^{7}$ Sin embargo, la evaluación del plexo submucoso requiere gran experiencia y los resultados no siempre son fiables. El hallazgo de un aumento significativo de fibras colinérgicas y adrenérgicas en las biopsias intestinales de esta enfermedad, ha permitido la incorporación de los métodos histoquímicos a las BRS, lo que representa un avance definitivo en el diagnóstico de las aganglionosis. ${ }^{\text {- }}$ 5, s-10, 14
Este trabajo refiere los resultados obtenidos con estudios histoquímicos de enzimas colinérgicas (Acetilcolinesterasa) en biopsias rectales por succión practicadas a 34 niños, con el fin de certificar o descartar el diagnóstico de aganglionosis intestinal congénita.

\section{MATERIAL Y METODO}

Entre octubre de 1979 y octubre de 1980 se realizaron BRS, utilizando el aparato de Ulrich, en 34 niños cuyas edades fluctuaban entre 1 mes y 12 años, y que hemos dividido en dos grupos:

- 12 niños operados por megacolon congénito.

- 22 niños controlados en la Policlínica de Gastroenterología Infantil del Hospital Gmo. Grant Benavente, de Concepción, por constipación crónica rebelde al tratamiento médico, con exámenes radiológicos $y / o$ manométricos sugerentes de megacolon.

La BRS se efectía en forma ambulatoria, previa preparación del niño con enemas evacuantes salinos, tomando dos muestras simultáneas a cinco y seis cm del ano.

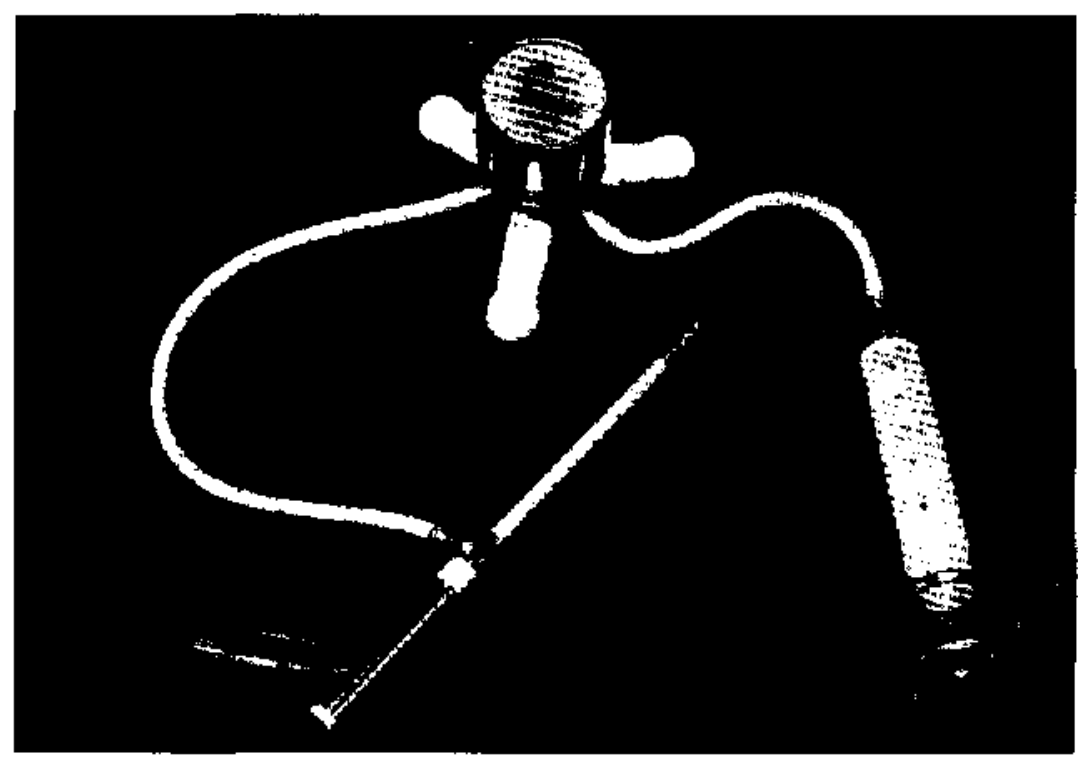

Figura 1. Aparato de Ulrich.

Las muestras se congelan con nieve carbónica y se almacenan en un refrigerador (Lo-Cold Freezer Scien. Temp.) a menos de 70 grados C. Posteriormente se hacen cortes de 14 micrones de espesor en un criostato (International Harris Cryos- tat) a menos de 20 grados, que se reciben sobre cubreobjetos y se dejan a temperatura ambiente por 15 a $20 \mathrm{~min}$. Se realizan tinciones con hernatoxilina-eosina para verificar la presencia de epitelio, tinica propia y muscular de la mucosa en cada 
una de las muestras. Para la determinación de actividad de la acetilcolinesterasa (ACE) se utiliza el método de KARNOWSKY-HOOTS modificado, incubando los cortes por 90 minutos a 37 grados C. 4, 5 La actividad del fermento se evidencia por tinción con color café de las estructuras que la contienen. El intestino normal adquiere una coloración moderada en el tejido muscular y en algunas fibras nerviosas de la submucosa, pero no en la lámina propia.

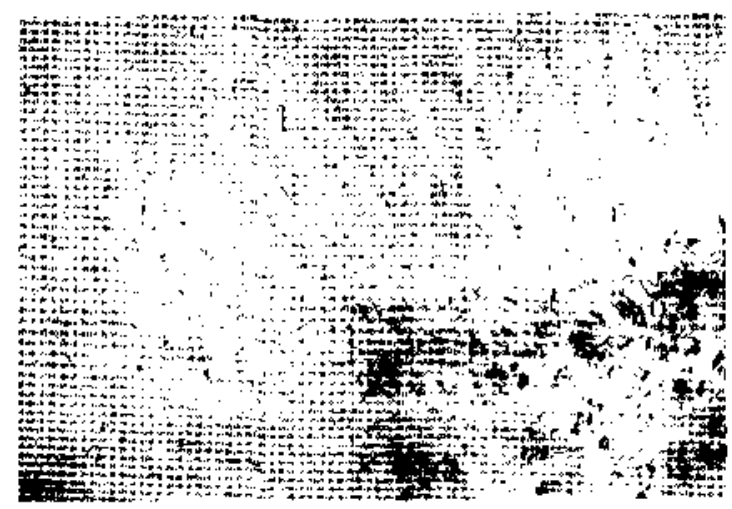

Figura 2. Mucosa rectal nomial. Tinción ACE. Se ubserva discretai ietividad ACE en la capa nuscular de la nucosa y ausercia de ella en la lámina propıa.

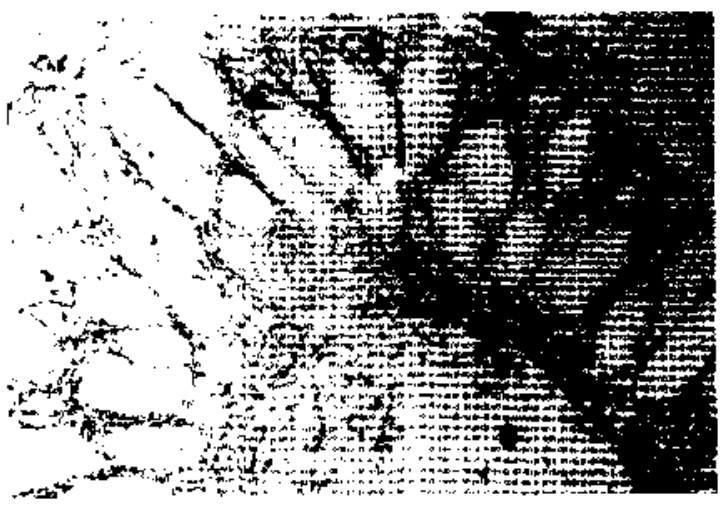

Figuras 3 y 4. Enfermedad de Hirschspnung. Tinción ACE. Hay intensa activiclirl enzimática en la linina propia. (Punteado oscuro.)

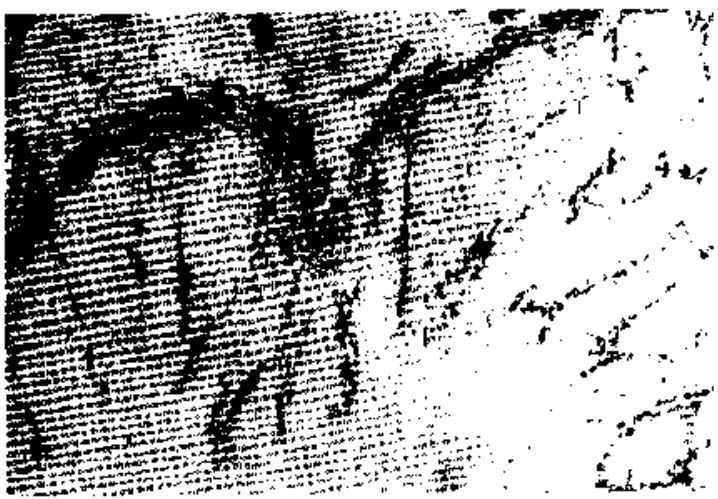

Solamente se consideran positivas las biopsias en que la actividad de ACE aparece muy aumentada en la túnica propia (entre las CRIPTAS) y en la muscular de la mucosa, que se ven intensamente teñidas.

\section{RESULTADOS}

En los 12 niños operados por megacolon congénito. se obtuvieron 7 biopsias con actividad ACE positiva, cinco de ellos tenían esfinteromiomectomía previa a la biopsia, por cuadros clínicos compatibles, manometrias alteradas y biopsias quirúrgicas con histología clásica, informadas como aganglionosis; los otros dos niños habian sido intervenidos por cuadros subobstructivos intestinales en el periodo de R.N. y actualmente están colostomizados. Cinco niños operados tienen biopsias con ACE negativa, cuatro de ellos fueron intervenidos con la técnica de Duhamel y la BRS se obturo desde el asa intestinal descendida, que es gangliónica. El otro niño con biopsia negativa se intervino por constipación intratable, teniendo manometria y biopsia histológica normales.

De las 22 biopsias en niños que consultaron en policlínica por constipación rebelde a tratamiento médico se obtuvieron 5 con ACE positiva: 2 fieron operados posteriormente y las biopsias quirúrgicas mostraron aganglionosis; dos no han vuelto a control y en un niño hay oposición paterna a la intervención propuesta.

De las 17 biopsias restantes con ACE (-), una fue tomada a un R.N. que presentaba distensión abdominal intensa con períodos de diarrea y constipación alternados, demostrándose posteriormente una intolerancia secundaria a la lactosa. Las otras 16 biopsias correspondieron a niños portadores de megacolon funcional, lo que fue corroborado por la evolución clínica y manometrías de control.

\section{COMENTARIO}

En el intestino normal, la actividad de la ACE se encuentra en el tejido muscular y en algunes fibras nerviosas de la submucosa, pero no ell el tejido intersticial de la mucosa. En cambio, en el intestino agangliónico de la enfermedad de Hirschsprung se comprueba un notorio aumento de ella en la musculatura, inciuyendo la muscular de la mucosa y una intensa actividad a nivel de la lámina propia, originada en el aumento de pro- 
ducción de la enzima por las fibrillas nerviosas que recorren el intersticio de la mucosa. Este fenómeno no ha sido descrito en otras enfermedades $y$ estas caracteristicas enzimáticas del recto aganglionar constituyen la base de un método de diagnóstico inocuo y altamente especifico. Las BRS con actividad ACE altamente positiva en lámina propia permiten por lo tanto reconocer la enfermedad de Hirschsprung. 1,2,4,5, y

En nuestro estudio observamos que en los niños operados hubo completa correlación entre la histopatología y la BRS. En los pacientes intervenidos con la técnica de Duhamel, la BRS se practicó después de la intervención, por lo que la muestra provenía del segmento intestinal descendido, cuya inervación es normal, resultando sin actividad ACE. De los niños con constipación, cinco tenian actividad ACE positiva; en los dos intervenidos posteriormente se demostró agangliosis en las biopsias histopatológicas, lo gue está de acuerdo con las descripciones de la literatura, al señalar que 8 a $10 \%$ del total de constipados rebeldes corresponden a aganglionosis congénitas con expresión clínica atenuada. ${ }^{1,25}$ Por último, los pacientes constipados funcionales demostraron, por su evolución clínica y manométrica, total concordancia con las BRS que mostraban ausencia de actividad ACE, reafirmando así el diagnóstico.

Por lo tanto, aunque el grupo de niños estudiados no es de gran tamaño, concluimos que el método de la ACE sirve para diferenciar los megacolon agangliónicos de los funcionales, y es fácil de realizar en manos de un equipo experto.

\section{RESUMEN}

Se realizan 34 BRS para estudio histoquímico de ACE, en niños portadores de enfermedad de Hirschsprung, operados y en constipados rebel- des a tratamiento médico. Se describe la técnica utilizada y los resultados obtenidos. Se concluye que es un método simple, rápido y útil para diagnosticar aganglionosis intestinal congénita.

\section{REFERENCLAS}

1 Martinez, C.; Amoyne, R.; Clover, M.: Monereo, J., y Contreras, F. Diugnóstico histoyurmicto de biopsias rectales por succion en la enlemedad de Hirschsprung. Rev. Clinica Españolá, T(5mo 150, Nos. 3-4, 1978, pp. $167-1 \overrightarrow{1} 1$.

2 Bneno Fadia. Fundamentos del diapónóstico histocuuimico de la exifermedad de Hirschsprung. Hev. Chil. Ped. Vol. $2 \overline{7}$, N.' 2,1976 , pp. 155-159.

3 Kehomald, M; Rupokn, J.; Louhimo, d. Diagnosis of Hirsehsprung disease. Acta Pediatr. Scand. 68: 893-97, 1979.

4 Chow, W.; Chan, W. C., and Paw C. K. Yul (Hong-Kong). Histochemical criteria for the diagnosis of Hirschsprung's disease in rectal suction biopsies by acetylcholinesterase activity. J Pediatr. Surg. Vol, 12, N, ${ }^{\circ} 5,1977.675-679$.

5 Meier Ruge, W.; Lutterbeck, P. M.; Herag, B.; Moryer, R.; Mocer, R., and Schânil, A Acetylcholinesterase activity in suction biopsies of the sectuni in diagnosis of Hirschsprung's disease. J. Pediatr. Surg. Vol. 1, X:. ${ }^{\circ}$ I, Febr. 1972, pp. 11-17.

6 Okamoto, Ebo, and Veda. Takachi. Einbryogenesis of intramural ganglia of the gut and its relation to Hirschspnung's disease. J. Pediatr. Surg. Vol. 2, N. ${ }^{\circ} 5$, Oct. 1967, pp. 437-443.

7 Compbell, P. E., and Noblett, Helen R. Experience with rectal suction biopsy in the diagnosis of Hirschsprung's disease. J. Pediatr. Surg. Vol. 4, N. 4,1969 , pp. $410-415$.

s Smith, d.; Bestey, G, and Parick W. Acetylcholinesterase in Hirschspnung's disease. Lancet $1(81 J 7)$ ): 665, marzo 1979.

9 Dode, $G$. et ats. Diagnostic value of rectal nucosal acetylcholinesterase levels in Hirschsprung's disease. Lancet 1 ( 8112 ) 347, Febr. 1979.

10 damet, J.; Howard, E.; Nixon, H. Histochernical diagnosis of Hirschspning's disease. Lancet $2(7617\})$ 436, Aug. 1969.

11 Passonge, Ebehard. The genetic of Hirschspning disease. New Engl. J. Med. 276: 138, 1967 .

12 Spanish Scandination Meeting Hirschsprung's disease: Scandinavian Association of Paediatric Surgeons. Acta Paediatr. Scand, 63: 307-319, 1974.

13 Gome, J. R.; Howard, E R., and Nixon, H. H. Autonomic nerves in rectum and colon in Hirschsprung's disease. A cholinesterase and catecholamine histoxhemical study. Arch. Dis. Childh., 44: 406, 1969.

14 Elema, J. P. Hirsctisprung's disedse: experience with sorre enzyme histochemical techniques. Arch. Dis. Childh., 48: 160,1973 . 